\begin{tabular}{|c|c|c|c|c|c|c|}
\hline \multirow{4}{*}{ Impact Factor: } & ISRA (India) & $=3.117$ & SIS (USA) & $=0.912$ & ICV (Poland) & $=6.630$ \\
\hline & ISI (Dubai, UAE & $=\mathbf{0 . 8 2 9}$ & РИНЦ (Russia & $=0.156$ & PIF (India) & $=1.940$ \\
\hline & GIF (Australia) & $=0.564$ & ESJI (KZ) & $=\mathbf{5 . 0 1 5}$ & IBI (India) & $=4.260$ \\
\hline & JIF & $=1.500$ & SJIF (Morocco & $=5.667$ & OAJI (USA) & $=0.350$ \\
\hline
\end{tabular}

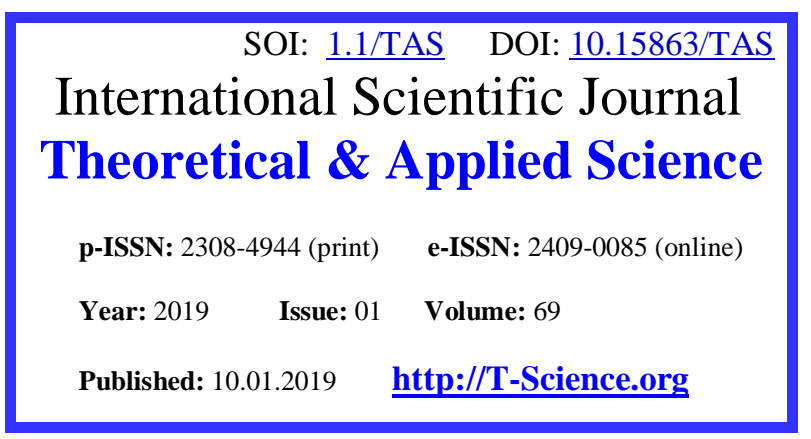

SECTION 2. Applied mathematics. Mathematical modeling.
QR - Issue

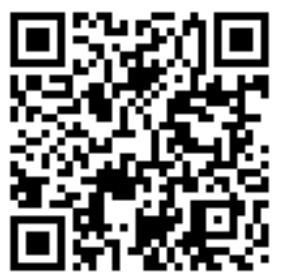

QR - Article

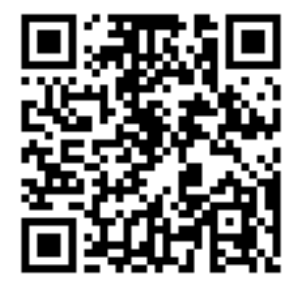

Gennady Evgenievich Markelov Candidate of Engineering Sciences, associate professor, corresponding member of International Academy of Theoretical and Applied Sciences, Bauman Moscow State Technical University, Moscow, Russia markelov@bmstu.ru

\title{
A WORKING MATHEMATICAL MODEL OF A TECHNICAL SYSTEM ELEMENT
}

Abstract: A working mathematical model of a technical system element was obtained. The technical system element includes a resistor with temperature-dependent resistance and total heat capacity. The created model is sufficiently full, accurate, adequate, productive, and economical. Such a mathematical model, when applied, requires less time and costs spent on research and enables efficient use of mathematical modeling tools.

Key words: working mathematical model, properties of mathematical models, principles of mathematical modeling.

Language: Russian

Citation: Markelov, G. E. (2019). A working mathematical model of a technical system element. ISJ Theoretical \& Applied Science, 01 (69), 52-55.

Soi: $\underline{\text { http://s-o-i.org/1.1/TAS-01-69-11 Doi: crossef https://dx.doi.org/10.15863/TAS.2019.01.69.11 }}$

\section{РАБОЧАЯ МАТЕМАТИЧЕСКАЯ МОДЕЛЬ ЭЛЕМЕНТА ТЕХНИЧЕСКОЙ СИСТЕМЫ}

Аннотация: Получена рабочая математическая модель элемента технической системы. Элемент технической системы включает резистор, сопротивление и полная теплоемкость которого зависят от температуры. Построенная модель в достаточной мере обладает свойствами полноты, точности, адекватности, продуктивности и экономичности. Применение такой математической модели приводит к сокращению затрат времени и средств на проведение исследования, позволяет рационально использовать возможности математического моделирования.

Ключевые слова: рабочая математическая модель, свойства математических моделей, приниипь математического моделирования.

\section{1. Введение}

Подходы к построению математических моделей различных технических систем изложены в обширной учебной и научной литературе. В работах $[1 ; 2]$ введено понятие рабочей математической модели и изложен единый подход к построению математической модели, которая в достаточной мере обладает нужными свойствами применительно к конкретному исследованию. Некоторые свойства математических моделей приведены в работах [3; 4]. В работе [5] рассмотрен пример построения математической модели, в достаточной мере обладающей нужными свойствами применительно к исследованию, некоторые результаты которого изложены в работах [6-8]. Особенности внедрения единого подхода к построению математических моделей рассмотрены в работах $[9 ; 10]$.

Целью настоящей работы является разработка в рамках единого подхода рабочей математической модели одного из элементов технической системы. Такой элемент включает резистор, сопротивление и полная теплоемкость которого зависят от температуры.

\section{2. Постановка задачи}

Резистор считаем высокотеплопроводным телом, температура $T$ которого в начальный момент времени $t_{0}$ равна $T_{0}$. На поверхности резистора площадью $S$ происходит 


\begin{tabular}{|c|c|c|c|c|c|c|}
\hline \multirow{4}{*}{ Impact Factor: } & ISRA (India) & $=3.117$ & SIS (USA) & $=0.912$ & ICV (Poland) & $=6.630$ \\
\hline & ISI (Dubai, UAE & $=0.829$ & РИНЦ (Russia & $=0.156$ & PIF (India) & $=1.940$ \\
\hline & GIF (Australia) & $=0.564$ & ESJI (KZ) & $=5.015$ & IBI (India) & $=4.260$ \\
\hline & JIF & $=1.500$ & SJIF (Morocco & $=5.667$ & OAJI (USA) & $=0.350$ \\
\hline
\end{tabular}

конвективный теплообмен с окружающей средой, температура которой равна $T_{0}$, коэффициент теплоотдачи известен и равен $\alpha$. Пусть

$$
\begin{aligned}
& R(T)=R_{0}\left[1+\beta\left(T-T_{0}\right)\right], \\
& C(T)=C_{0}\left[1+\gamma\left(T-T_{0}\right)\right],
\end{aligned}
$$

где $R(T)$ и $C(T)$ - сопротивление и полная теплоемкость резистора; $R_{0}$ и $C_{0}-$ сопротивление и полная теплоемкость резистора при $T=T_{0} ; \quad \beta \quad$ и $\quad \gamma \quad$ температурные коэффициенты, причем $\beta>0$ и $\gamma>0$. Через резистор протекает электрический ток, сила которого равна

$$
I=\frac{U}{R_{0}\left[1+\beta\left(T-T_{0}\right)\right]},
$$

где $U-$ постоянная разность электрических потенциалов на полюсах рассматриваемого элемента.

В рамках проводимого исследования представляет интерес величина $I$. Построим рабочую математическую модель объекта исследования, которая в достаточной мере обладает свойствами полноты, точности, адекватности, продуктивности и экономичности.

\section{3. Решение задачи}

Для решения поставленной задачи выстроим иерархию математических моделей данного объекта исследования и определим условия, при выполнении которых можно с относительной погрешностью не более заданного значения $\delta_{0}$ найти искомую величину $I$.

Если разность $T-T_{0}$ достаточно мала, то согласно (1) найдем искомую величину по формуле

$$
I_{0}=U / R_{0} .
$$

Определим условия, при которых применима полученная формула. Для этого рассмотрим установившийся процесс теплообмена. В этом случае мощность тепловыделения в материале резистора равна тепловому потоку, отводимому от резистора, т. е.

$$
\frac{U^{2}}{R_{0}\left[1+\beta\left(T_{*}-T_{0}\right)\right]}=\alpha\left(T_{*}-T_{0}\right) S,
$$

где $T_{*}$ - установившееся значение температуры резистора. Из полученного равенства легко найти

$$
T_{*}=T_{0}+\frac{1}{2 \beta}\left(-1+\sqrt{1+\frac{4 \beta U^{2}}{\alpha S R_{0}}}\right),
$$

а затем определить установившееся значение

$$
I_{*}=\frac{U}{R\left(T_{*}\right)}=\frac{2 I_{0}}{1+\sqrt{1+4 \beta U I_{0} /(\alpha S)}} .
$$

Очевидно, что $\quad I_{*} \leq I \leq I_{0} . \quad$ Тогда для относительной погрешности величины $I_{0}$ запишем

$$
\delta\left(I_{0}\right)=\left|\frac{I-I_{0}}{I}\right|=\frac{I_{0}}{I}-1 \leq \frac{I_{0}}{I_{*}}-1 .
$$

Следовательно, при выполнении условия

$$
\frac{I_{0}}{I_{*}}-1 \leq \delta_{0}
$$

можно с относительной погрешностью не более $\delta_{0}$ использовать формулу (2) для нахождения искомой величины. Тогда приходим к неравенству

$$
\frac{\beta U I_{0}}{\alpha S} \leq\left(\delta_{0}+1\right) \delta_{0} .
$$

При выполнении этого неравенства математическая модель (2) в достаточной мере обладает свойствами полноты, точности, адекватности, продуктивности и экономичности.

Определим условия, при которых применима математическая модель (3). Для этого рассмотрим неустановившийся процесс теплообмена. В этом случае изменение температуры резистора во времени $t$ описывает обыкновенное дифференциальное уравнение первого порядка

$$
C(T) \frac{d T}{d t}=\frac{U^{2}}{R(T)}-\alpha\left(T-T_{0}\right) S,
$$

а начальное условие имеет вид

$$
T\left(t_{0}\right)=T_{0} \text {. }
$$

Учитывая, что

$$
I=\frac{I_{0}}{1+\beta\left(T-T_{0}\right)},
$$

сформулируем задачу Коши

$$
\begin{aligned}
& \frac{d I}{d t}=\frac{\beta I^{2}}{C_{0} I_{0}} \frac{\alpha S\left(I_{0}-I\right)-\beta U I^{2}}{\gamma\left(I_{0}-I\right)+\beta I}, \\
& I\left(t_{0}\right)=I_{0} .
\end{aligned}
$$

При выполнении условия

$$
\delta\left(I_{*}\right)=\left|\frac{I-I_{*}}{I}\right|=1-\frac{I_{*}}{I} \leq \delta_{0}
$$

можно с относительной погрешностью не более $\delta_{0}$ использовать формулу (3) для нахождения искомой величины, причем

$$
\delta_{0}<\frac{I_{0}}{I_{*}}-1,
$$

так как в противном случае следует применять формулу (2). Затем найдем момент времени

$$
\begin{aligned}
t_{*}=t_{0}+ & \frac{C_{0}}{\alpha S}\left[\frac{\gamma}{\beta}\left(\frac{I_{*}}{I_{0}}-1+\delta_{0}\right) \frac{I_{0}}{I_{*}}+\left(\frac{I_{0}}{2 I_{0}-I_{*}}+\right.\right. \\
& \left.+\frac{\gamma}{\beta} \frac{I_{0}-I_{*}}{2 I_{0}-I_{*}} \frac{I_{0}}{I_{*}}-1\right) \ln \left(2-\frac{I_{*}}{I_{0}}-\delta_{0}\right)-
\end{aligned}
$$




\begin{tabular}{|c|c|c|c|c|c|c|}
\hline \multirow{4}{*}{ Impact Factor: } & ISRA (India) & $=3.117$ & SIS (USA) & $=0.912$ & ICV (Poland) & $=6.630$ \\
\hline & ISI (Dubai, UAE & $=0.829$ & РИНЦ (Russia & $=0.156$ & PIF (India) & $=1.940$ \\
\hline & GIF (Australia) & $=0.564$ & ESJI (KZ) & $=\mathbf{5 . 0 1 5}$ & IBI (India) & $=4.260$ \\
\hline & JIF & $=1.500$ & SJIF (Morocco & $=5.667$ & OAJI (USA) & $=0.350$ \\
\hline
\end{tabular}

$$
\left.-\left(\frac{I_{0}}{2 I_{0}-I_{*}}+\frac{\gamma}{\beta} \frac{I_{0}-I_{*}}{2 I_{0}-I_{*}} \frac{I_{0}}{I_{*}}\right) \ln \left(\frac{I_{0}}{I_{0}-I_{*}} \delta_{0}\right)\right],
$$

для которого $I\left(t_{*}\right)=I_{*} /\left(1-\delta_{0}\right)$. Тогда согласно

(5) установившееся значение $I_{*}$ можно $\mathrm{c}$ относительной погрешностью не более $\delta_{0}$ считать равным $I(t)$ при $t \geq t_{*}$.

Если не выполнено условие (4), то математическая модель (3) при $t \geq t_{*}$ в достаточной мере обладает свойствами полноты, точности, адекватности, продуктивности и экономичности.

Разработка новой математической модели при формировании иерархии математических моделей объекта исследования может привести к уточнению найденных ранее условий применимости построенных математических моделей. Действительно, используя математическую модель (5), можно уточнить условие применимости формулы (2). Для этого найдем момент времени

$$
\begin{aligned}
t^{*}= & t_{0}+\frac{C_{0}}{\alpha S}\left[\left(\frac{I_{0}}{2 I_{0}-I_{*}}+\right.\right. \\
& \left.+\frac{\gamma}{\beta} \frac{I_{0}-I_{*}}{2 I_{0}-I_{*}} \frac{I_{0}}{I_{*}}-1\right) \ln \left(1+\frac{I_{*}}{I_{0}} \delta_{0}\right)-\frac{\gamma}{\beta} \delta_{0}- \\
& \left.-\left(\frac{I_{0}}{2 I_{0}-I_{*}}+\frac{\gamma}{\beta} \frac{I_{0}-I_{*}}{2 I_{0}-I_{*}} \frac{I_{0}}{I_{*}}\right) \ln \left(1-\frac{I_{*}}{I_{0}-I_{*}} \delta_{0}\right)\right],
\end{aligned}
$$

для которого $I\left(t^{*}\right)=I_{0} /\left(1+\delta_{0}\right)$. Тогда значение $I_{0}$ можно с относительной погрешностью не более $\delta_{0}$ считать равным $I(t)$ при $t \leq t^{*}$.
Если выполнено условие (4) или $t \leq t^{*}$, то математическая модель (2) в достаточной мере обладает свойствами полноты, точности, адекватности, продуктивности и экономичности.

\section{4. Результаты}

Построение иерархии математических моделей позволяет выявить рабочую математическую модель, которая в достаточной мере обладает нужными свойствами применительно к конкретному исследованию. Действительно, если выполняется неравенство (4) или в рамках проводимого исследования $t \leq t^{*}$, то математическую модель (2) считаем рабочей. Если не выполнено условие (4), а временной интервал от $t_{0}$ до $t_{*}$ можно в рамках проводимого исследования не рассматривать, то выбираем математическую модель (3) как рабочую, иначе - математическую модель (5).

\section{5. Заключение}

Таким образом, в рамках единого подхода сформулированы утверждения, которые позволяют установить рабочую математическую модель элемента технической системы. Построенная математическая модель в достаточной мере обладает свойствами полноты, точности, адекватности, продуктивности и экономичности применительно к данному исследованию.

Очевидно, что применение такой модели приводит к сокращению затрат времени и средств на проведение исследования, позволяет рационально использовать возможности математического моделирования.

\section{References:}

1. Markelov, G. E. (2015). On Approach to Constructing a Working Mathematical Model. ISJ Theoretical \& Applied Science, 04 (24): 287-290. Soi: http://s-oi.org/1.1/TAS*04(24)52

Doi: http://dx.doi.org/10.15863/TAS.2015.04.24.52

2. Markelov, G. E. (2015). Constructing a Working Mathematical Model. ISJ Theoretical \& Applied Science, 08 (28): 44-46. Soi: http://s-o-i.org/1.1/TAS-08-28-6 Doi: http://dx.doi.org/10.15863/TAS.2015.08.28.6

3. Myshkis, A. D. (2011). Elements of the Theory of Mathematical Models [in Russian]. Moscow: URSS.
4. Zarubin, V. S. (2010). Mathematical Modeling in Engineering [in Russian]. Moscow: Izd-vo MGTU im. N.E. Baumana.

5. Markelov, G. E. (2012). Peculiarities of Construction of Mathematical Models. Inzhenernyi zhurnal: nauka i innovatsii, No. 4, http://engjournal.ru/catalog/mathmodel/hidden/ $\underline{150 . h t m l}$

6. Markelov, G. E. (2000). Effect of initial heating of the jet-forming layer of shaped-charge liners on the ultimate elongation of jet elements. $J$. Appl. Mech. and Tech. Phys., 41, No. 2, 231234. 


\begin{tabular}{|c|c|c|c|c|c|c|}
\hline \multirow{4}{*}{ Impact Factor: } & ISRA (India) & $=3.117$ & SIS (USA) & $=0.912$ & ICV (Poland) & $=6.630$ \\
\hline & ISI (Dubai, UAE & $=0.829$ & РИНЦ (Russia & $=0.156$ & PIF (India) & $=1.940$ \\
\hline & GIF (Australia) & $=0.564$ & ESJI (KZ) & $=\mathbf{5 . 0 1 5}$ & IBI (India) & $=4.260$ \\
\hline & JIF & $=1.500$ & SJIF (Morocco & $=5.667$ & OAJI (USA) & $=0.350$ \\
\hline
\end{tabular}

7. Markelov, G. E. (2000). Effect of initial heating of shaped charge liners on shaped charge penetration. J. Appl. Mech. and Tech. Phys., 41, No. 5, 788-791.

8. Markelov, G. E. (2000). Influence of heating temperature on the ultimate elongation of shaped-charge jet elements. Proc. of the 5th Int. Conf. "Lavrentyev Readings on Mathematics, Mechanics and Physics". (p.170). Novosibirsk: Lavrentyev Institute of Hydrodynamics.

9. Markelov, G. E. (2015). Particular Aspects of Teaching the Fundamentals of Mathematical
Modeling. ISJ Theoretical \& Applied Science, 05 (25), 69-72.

Soi: http://s-o-i.org/1.1/TAS*05(25)14 Doi: http://dx.doi.org/10.15863/TAS.2015.05.25.14

10. Markelov, G. E. (2016). Teaching the Basics of Mathematical Modeling. Part 2. ISJ Theoretical \& Applied Science, 01 (33), 72-74. Soi: http://s-o-i.org/1.1/TAS-01-33-15 Doi: http://dx.doi.org/10.15863/TAS.2016.01.33.15 